\title{
Hierarchy in the Phase Space and Dark Matter Astronomy
}

\author{
Niayesh Afshordi, ${ }^{1,2}$ 月 Roya Mohayaee, ${ }^{3}$ and Edmund Bertschinger ${ }^{4}$ \\ ${ }^{1}$ Perimeter Institute for Theoretical Physics, 31 Caroline St. N., Waterloo, ON, N2L 2Y5, Canada \\ ${ }^{2}$ Department of Physics and Astronomy, University of Waterloo, \\ 200 University Avenue West, Waterloo, ON, N2L 3G1, Canada \\ ${ }^{3}$ Institut d'Astrophysique de Paris, CNRS, UPMC, 98 bis boulevard Arago, Paris, France \\ ${ }^{4}$ Department of Physics and Kavli Institute for Astrophysics and Space Research, MIT \\ Room 37-602A, 77 Massachusetts Ave., Cambridge, MA 02139, USA
}

(Dated: October 26, 2018)

\begin{abstract}
We develop a theoretical framework for describing the hierarchical structure of the phase space of cold dark matter haloes, due to gravitationally bound substructures. Because it includes the full hierarchy of the cold dark matter initial conditions and is hence complementary to the halo model, the stable clustering hypothesis is applied for the first time here to the small-scale phase space structure. As an application, we show that the particle dark matter annihilation signal could be up to two orders of magnitude larger than that of the smooth halo within the Galactic virial radius. The local boost is inversely proportional to the smooth halo density, and thus is $\mathcal{O}(1)$ within the solar radius, which could translate into interesting signatures for dark matter direct detection experiments: The temporal correlation of dark matter detection can change by a factor of 2 in the span of 10 years, while there will be significant correlations in the velocity space of dark matter particles. This can introduce $\mathcal{O}(1)$ uncertainty in the direction of local dark matter wind, which was believed to be a benchmark of directional dark matter searches or the annual modulation signal.
\end{abstract}

Among the favorite dark matter candidates are the weakly interacting massive particles (WIMP), yet to be detected in particle accelerators. Different experiments look for a signature of WIMPs directly as they pass through Earth and recoil off the atomic nuclei in laboratory detectors, or indirectly through the byproducts of their self-annihilation into standard model particles (such as photons, electron/positron pairs, or neutrinos). The cross-section for the (s-wave) self-annihilation is also fixed by the relic abundance: $\left\langle\sigma_{\text {ann }} v\right\rangle \sim 10^{-31}$ $10^{-26} \mathrm{~cm}^{3} / \mathrm{s}$. Therefore, one would expect an astrophysical luminosity of:

$$
\frac{d L_{i}}{d E_{i}}=\frac{\left\langle\sigma_{\text {ann }} v\right\rangle E_{i}}{2 m_{\chi}^{2}} \frac{d B_{i}}{d E_{i}} \Phi ; \quad \Phi \equiv \int \rho^{2}(\mathbf{x}) d^{3} \mathbf{x}
$$

where $m_{\chi}$ is the WIMP mass, $E_{i}$ is the energy of the byproducts, $d B_{i} / d E_{i}$ is the differential branching ratio into $i\left(=\gamma, e^{+} e^{-}\right.$or $\left.\nu \bar{\nu}\right)$ particles and $\Phi$ is fixed by the spatial density distribution of dark matter within the emitting region.

All the factors in (1), except for $\Phi$, are fixed by the particle physics model (e.g. [1]). Unfortunately the gravitational potential of dark matter haloes says little about the contribution of small scale structure to $\Phi$. The cold dark matter $(\mathrm{CDM})$ primordial power spectrum predicts a large range of mass scales, from $10^{12}-10^{14} M_{\odot}$ down to $10^{-12}-10^{-4} M_{\odot}$ [2], far below the resolution limit of the present-day simulations at $z=0$, which is at best $10^{4} M_{\odot}$. In fact, simulations already see a nearly constant contribution to $\Phi$ per decade in sub-structure mass [3, 田, suggesting a significant contribution from unresolved structures.

Extrapolation of the simulated properties of the subhaloes to below the resolution limit, as well as assump- tions about the spatial/mass dependence of the boost due to sub-structure (e.g. [5, 6, 7]) could lead to significant over/underestimations (especially for a non-scaleinvariant linear power spectrum such as CDM). Similarly, the assumption of Maxwell-Boltzmann velocity distribution in direct detection experiments of dark matter particles could miss important phenomenological signatures of clustering in the phase space.

In this letter, we use the stable clustering hypothesis in order to predict the clustering of dark matter particles in the phase space, and consider the implications for indirect and direct dark matter searches. The stable clustering hypothesis was first introduced by Davis \& Peebles [8] as an analytic technique to study the galaxy correlation function in the deeply non-linear regime, and was subsequently applied into fitting formulae for non-linear correlation functions (e.g. 9, 10, 11)). The hypothesis assumes that the number of neighbors within a fixed physical separation becomes a constant (or pairwise velocity vanishes) on small scales, when the non-linear structure formation is completed. However, it became clear that this cannot be a good approximation on the scale of virial radius of CDM haloes ( $\sim 1 \mathrm{Mpc}$ today) as halo mergers and the subsequent tidal disruption can dissolve the old structures into newly formed haloes (e.g. [12, 13]). Nevertheless, the alternative analytic framework, known as the halo model, which has been extensively used in the literature over the past decade, misses the small scale structure of the haloes (i.e. sub-haloes, sub-sub-haloes, etc.) that do naturally form in hierarchical structure formation, and are ubiquitous in high resolution N-body simulations (e.g. 四).

In contrast, the merging and tidal activity should cease at scales much smaller than the virial radius of CDM 
haloes, suggesting that the stable clustering regime might be achieved on small enough scales. In other words, while sub-haloes could lose a large fraction of their mass due to tidal heating/stripping, a small fraction could remain gravitationally bound (e.g. [14]). As the mean/virial density of haloes drops as $1 /$ time $^{2}$, the gravitationally bound remnant will eventually become resilient to tidal disturbances. The stable clustering hypothesis can be trivially extended to the phase space where similar to real space it would predict that the number of particles within the physical velocity $\Delta \mathbf{v}$ and physical distance $\Delta \mathbf{r}$ of a given particle does not change with time for small enough $\Delta \mathbf{v}$ and $\Delta \mathbf{r}$.

In order to develop the stable clustering formalism in phase space, we start with the collisionless Boltzmann equation at the phase space coordinates, $\mathbf{r}+\Delta \mathbf{r}, \mathbf{v}+\Delta \mathbf{v}$, i.e.

$$
\begin{aligned}
& \frac{d f}{d t}(\mathbf{r}+\Delta \mathbf{r}, \mathbf{v}+\Delta \mathbf{v}, t)= \\
& \frac{\partial f}{\partial t}+\frac{\partial f}{\partial \mathbf{r}} \cdot(\mathbf{v}+\Delta \mathbf{v})-\frac{\partial f}{\partial \mathbf{v}} \cdot(\nabla \phi+\nabla \nabla \phi \cdot \Delta \mathbf{r})=0 .
\end{aligned}
$$

The above equation in terms of the new function

$$
\tilde{f}_{i}(\Delta \mathbf{r}, \Delta \mathbf{v}) \equiv f\left(\mathbf{r}_{i}+\Delta \mathbf{r}, \mathbf{v}_{i}+\Delta \mathbf{v}\right)
$$

for particle $i$, can be re-written as

$$
\frac{d f}{d t}=\left.\frac{\partial \tilde{f}_{i}}{\partial t}\right|_{\Delta \mathbf{r}, \Delta \mathbf{v}}+\frac{\partial \tilde{f}_{i}}{\partial \Delta \mathbf{r}} \cdot \Delta \mathbf{v}-\frac{\partial \tilde{f}_{i}}{\partial \Delta \mathbf{v}} \cdot(\nabla \nabla \phi \cdot \Delta \mathbf{r})=0
$$

where

$$
\left.\frac{\partial \tilde{f}_{i}}{\partial t}\right|_{\Delta \mathbf{r}, \Delta \mathbf{v}}=\frac{\partial \tilde{f}_{i}}{\partial t}+\mathbf{v} \cdot \frac{\partial \tilde{f}_{i}}{\partial \Delta \mathbf{r}}-\nabla \phi \cdot \frac{\partial \tilde{f}_{i}}{\partial \Delta \mathbf{v}}
$$

The stable clustering hypothesis assumes that the above expression when averaged over the particles vanishes for small $\Delta r$ and $\Delta v$. If we assume that $\left\langle\tilde{f}_{i} \nabla \nabla \phi\right\rangle_{p} \approx$ $\left\langle\tilde{f}_{i}\right\rangle_{p}\langle\nabla \nabla \phi\rangle_{p}$ then a solution to (耳), averaged over particles is

$$
\langle\tilde{f}\rangle_{p} \equiv \frac{1}{N} \sum_{i} \tilde{f}_{i}=F\left[\Delta \mathbf{v}^{2}+\Delta x_{j} \Delta x_{k}\left\langle\partial_{j} \partial_{k} \phi\right\rangle_{p}\right]
$$

which is the most general time-independent solution with an isotropic velocity distribution, where $F$ is an arbitrary function, and $N$ is the number of particles in the phasespace volume of interest. We later show that averaging over particles and volume-averaging differ only by a constant. Next, we use the approximation that the potential is spherically symmetric and hence the above solution, using Poisson equation, can be re-written as

$$
\langle\tilde{f}\rangle_{p}=\mu \xi_{s}=F\left[(\Delta \mathbf{v})^{2}+100 H\left(\xi_{s}\right)^{2}(\Delta \mathbf{r})^{2}\right]
$$

where $\xi_{s}$ is the phase-space density at the formation time of the sub-structure, and we have used the fact that the post-collapse halo density is roughly $\sim 200$ times the critical density at the formation time [15]. $\mu \sim 1-10 \%$ is the mean fraction of bound particles that can survive the tidal disruption period.

In order to find the function $F$, we use the spherical collapse results

$$
\xi_{s} \sim \frac{10 H\left(\xi_{s}\right)}{G^{2} M\left(\xi_{s}\right)}
$$

and also that the radius and velocity dispersion of haloes are related by (e.g. [16]): $\sigma_{\text {vir }} \sim 10 H r_{\text {vir }}$, where $H$ is the Hubble constant at the time of halo's collapse. Hence (8) is roughly the phase space density of haloes that collapse at Hubble constant $H\left(\xi_{s}\right)$ and mass $M\left(\xi_{s}\right)$. The phase space volume of the collapsed halo, i.e. the volume of the constant- $\xi_{s}$ ellipsoid in (7) is $M / \xi_{s}$ and using (8) we have:

$$
\left[\frac{\pi F^{-1}\left(\mu \xi_{s}\right)}{10 H\left(\xi_{s}\right)}\right]^{3}=\frac{\left[G M\left(\xi_{s}\right)\right]^{2}}{10 H\left(\xi_{s}\right)} .
$$

Furthermore, the mass scale that collapses at a given cosmological epoch is characterized by:

$$
\left[\frac{H\left(\xi_{s}\right)}{H_{0}}\right]^{-2 / 3} \sigma\left[M\left(\xi_{s}\right)\right] \sim \delta_{c} \simeq 1.7
$$

where $\delta_{c}$ is the linear density threshold for the spherical collapse, while $\sigma[M]$ is the r.m.s. top-hat linear overdensity at the mass scale $M$, and $H_{0}$ is the present day Hubble constant. We remark that the effect of dark energy will be a constant factor that could be absorbed in the definition of $\mu$, since most sub-haloes have formed long before the era of dark energy dominance.

Using the above results, the phase space correlation function takes the form:

$$
\begin{aligned}
& \left\langle f\left(\mathbf{r}_{1}, \mathbf{v}_{1}\right) f\left(\mathbf{r}_{2}, \mathbf{v}_{2}\right)\right\rangle \\
& \simeq \frac{1}{V_{6}} \int_{V_{6}} d^{3} r d^{3} v f(\mathbf{r}, \mathbf{v}) f(\mathbf{r}+\Delta \mathbf{r}, \mathbf{v}+\Delta \mathbf{v}) \\
& =\frac{1}{V_{6}} \sum_{i} f\left(\mathbf{r}_{i}+\Delta \mathbf{r}, \mathbf{v}_{i}+\Delta \mathbf{v}\right)=\frac{N}{V_{6}}\langle\tilde{f}\rangle_{p} \\
& \simeq\left\langle f\left(\mathbf{r}_{1}, \mathbf{v}_{1}\right)\right\rangle\left\langle f\left(\mathbf{r}_{2}, \mathbf{v}_{2}\right)\right\rangle+\mu\langle f(\overline{\mathbf{r}}, \overline{\mathbf{v}})\rangle \xi_{s}(\Delta \mathbf{r}, \Delta \mathbf{v}),
\end{aligned}
$$

where we used the assumption of ergodicity to replace the ensemble average \langle\rangle by the volume average, in a given volume of the phase space $V_{6}$, while $(\overline{\mathbf{r}}, \overline{\mathbf{v}})$ are the mean values of $\left(\mathbf{r}_{1}, \mathbf{v}_{1}\right)$ and $\left(\mathbf{r}_{2}, \mathbf{v}_{2}\right)$. The second term in Eq. (12) dominates in the stable clustering regime, where $|\Delta \mathbf{v}|=\left|\mathbf{v}_{1}-\mathbf{v}_{2}\right| \ll \Delta v_{\text {tid }}$ and $|\Delta \mathbf{r}|=\left|\mathbf{r}_{1}-\mathbf{r}_{2}\right| \ll \Delta r_{\text {tid }}$, and $\Delta v_{\text {tid }}$ and $\Delta r_{\text {tid }}$ characterize the tidal truncation radius in the phase space. On the other hand, the first term dominates Eq. (12) for large separations in the phase space, where particles are not correlated. In other 
words, Eq. (12) is an interpolation between the stable clustering and the smooth halo regimes.

The annihilation signal from gravitationally bound substructure is found by integrating (12) within the phase-space stable clustering hypothesis:

$$
\begin{aligned}
\delta \Phi_{\text {sub. }} & \simeq \int d^{3} \mathbf{x} \int d^{3} \mathbf{v}_{1} d^{3} \mathbf{v}_{2}\left\langle f\left(\mathbf{x}, \mathbf{v}_{1}\right)\right\rangle \mu \xi_{s}\left(0, \mathbf{v}_{\mathbf{1}}-\mathbf{v}_{\mathbf{2}}\right) \\
& =\int d^{3} \mathbf{x} \rho_{\text {halo }}(\mathrm{x}) \int d^{3} \boldsymbol{\Delta} \mathbf{v} \cdot \mu \xi_{s}(0, \boldsymbol{\Delta} \mathbf{v})
\end{aligned}
$$

yielding the local boost factor of :

$$
\begin{aligned}
& B_{\text {sub. }}(\mathrm{x}) \equiv \frac{\left\langle\delta \rho_{\text {halo }}(\mathrm{x})^{2}\right\rangle}{\left\langle\rho_{\text {halo }}(\mathrm{x})\right\rangle^{2}} \simeq \frac{\mu}{\rho_{\text {halo }}(\mathrm{x})} \int d^{3} \boldsymbol{\Delta} \mathbf{v} \cdot \xi_{s}(0, \boldsymbol{\Delta} \mathbf{v}) \\
& \simeq \frac{8 \pi^{1 / 2} \mu}{9 \delta_{c}^{3}}\left(\frac{\rho_{\text {halo }}(\mathrm{x})}{200 \rho_{\text {crit }, 0}}\right)^{-1} \int_{M_{\min }}^{M_{\max }} M^{-2} d\left[M^{2} \sigma^{3}(M)\right]
\end{aligned}
$$

where we have used the stable clustering framework developed in (7)-(10) to substitute for $\xi_{s}$.

The asymptotic form of CDM linear power spectrum on small scales is (e.g. 17) $P(k) \propto k^{n_{s}-4} \ln ^{2}\left(k / k_{\text {eq }}\right)$, where $n_{s}$ is the primordial adiabatic scalar index, and $k_{\text {eq }} \simeq 0.56 \Omega_{m} h^{2} \mathrm{Mpc}^{-1}$ is related to the comoving scale of the horizon at matter-radiation equality. Using this asymptotic form, we can find an analytic approximation for the boost factor:

$$
\begin{gathered}
B_{\text {sub. }}(\mathbf{x})=\mu\left(\frac{\rho_{\text {halo }}(\mathbf{x})}{200 \rho_{\text {crit }, 0}}\right)^{-1}\left(\frac{\Omega_{m} h}{0.27 \times 0.7}\right)^{\frac{3}{2}\left(n_{\text {eff }, 8}+3\right)} \\
\times\left(\frac{\sigma_{8}}{0.8}\right)^{3}\left\{K\left[\ln ^{1 / 2}\left(\frac{M_{\text {eq }}}{M_{\text {min }}}\right)\right]-K\left[\ln ^{1 / 2}\left(\frac{M_{\text {eq }}}{M_{\text {max }}}\right)\right]\right\}(15)
\end{gathered}
$$

where $M_{\text {eq }}=\left(2.9 \times 10^{14} M_{\odot}\right)\left(\Omega_{m} h^{2}\right)^{-2}$, is the mass associated with the horizon scale at matter-radiation equality, $n_{\mathrm{eff}, 8} \simeq-1.68$, is the logarithmic slope of the linear power spectrum at $\sim 8 h^{-1} \mathrm{Mpc}$, and

$$
K(y) \simeq \frac{9}{10^{5}}\left(\frac{4 y^{11}}{11}-y^{9}\right) \exp \left[\frac{1}{2}\left(y^{2}-16\right)\left(n_{s}-1\right)\right]
$$

This analytic approximation (15) is within $40 \%$ of the exact integral in (14) for $10^{-15} M_{\odot}<M<10^{10} M_{\odot}$ and $0.95<n_{s}<1.05$ (where we compare with the fitting form of 18 for the CDM power spectrum). For supersymmetric dark matter models, the minimum $\mathrm{CDM}$ halo mass can range from $10^{-12} M_{\odot}-10^{-4} M_{\odot}$, yielding $K \simeq 10^{5}$ within a factor of 3 .

To calibrate the parameter $\mu$, we can compare our results to numerical simulations. Matching the expected boost per mass decade to that of "Via Lactea" mock Milky Way simulation [3], which is around 0.1 for subhalo masses $10^{7} M_{\odot}<M_{\text {sub }}<10^{10} M_{\odot}$, with their assumed cosmology, yields $\mu \simeq 0.026$, which is comparable to the fraction of simulated halo mass found in (resolved) gravitationally bound sub-haloes (e.g. $5.3 \%$ in [3]).

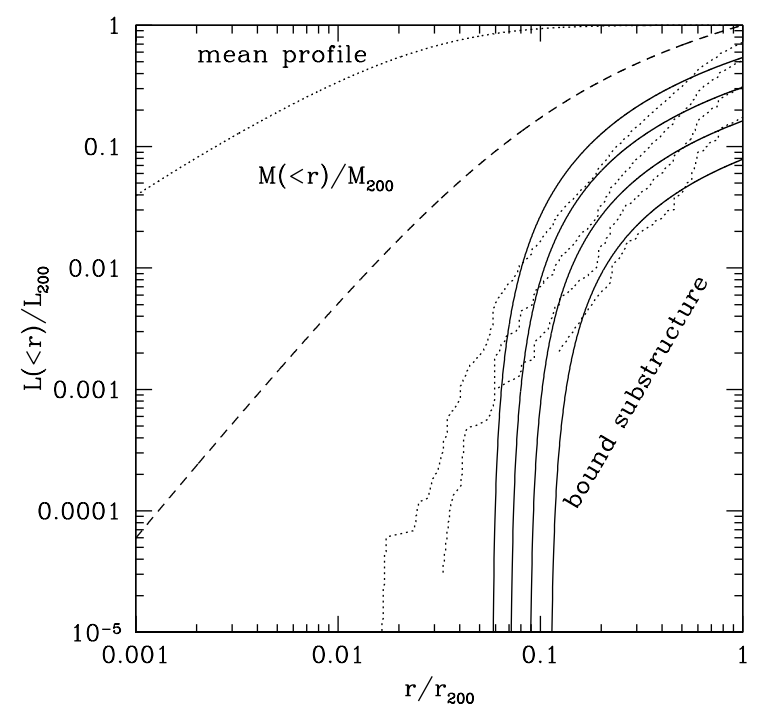

FIG. 1: The cumulative radial profile of DM annihilation: top dotted curve shows the contribution from the mean profile, while the lower dotted curves show the simulated contribution of sub-haloes more massive than $10^{5,6,7,8} M_{\odot}$. The solid curves show our analytic prediction, with the same cut-off's on $M_{\text {min }}$, and a tidal cut-off at the high mass end. The dashed curve shows the cumulative mass profile for comparison.

Fig.(11) compares the prediction for the annihilation profile (solid curves) with numerical simulations of [4] (dotted curves), which shows reasonable agreement. To do this, we have cut off the integral (14) at high masses by requiring that the formation density of bound subhaloes must be lower than the local halo density. The crudeness of this criterion is most likely responsible for the sharp drop in the signal at small radii, relative to the numerical results. However, we note that (surviving) sub-solar mass subhaloes, that dominate the boost for realistic WIMP models, are much less affected by the tidal effects of the host halo (which was the motivation for the stable clustering approximation). Moreover, the simulated results do not include the effect of sub-sub-haloes, etc. which could be another source of difference with our prediction.

Fig. (2) shows that not only the CDM hierarchy can significantly boost the total annihilation signal from the Milky Way (and dark matter haloes in general), they can also affect the local density variance at the solar radius $\left(B_{\text {sub. }} \sim 2-6\right)$, which can have interesting implications for direct dark matter detection searches. We can quantify the latter through the temporal correlation of dark matter detection signal $D(t)$, which traces the local density of the dark matter halo at the solar system. For simplicity, we will assume that the solar system is moving through the CDM hierarchy at $v \simeq \sqrt{2.5} \times 250 \mathrm{~km} / \mathrm{s}$ (assuming a singular isothermal mean phase space dis- 


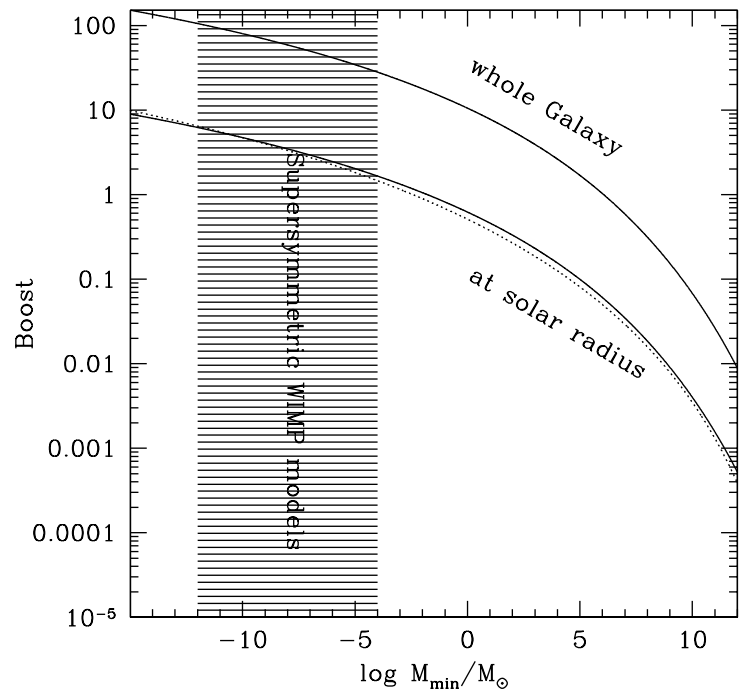

FIG. 2: Boost to the dark matter annihilation signal due to substructure with the formation mass larger than $M_{\min }$. The lower solid curve is the local boost factor at solar radius (assuming $\rho=10^{5} \rho_{\text {crit }}$ ), while the upper solid curve shows the mean boost estimated for the whole Milky Way halo (out to 16 times NFW scale radius [3]), where we have assumed $n_{s}=0.96, \sigma_{8}=0.82, \Omega_{m}=0.28$, and $h=0.7$. The dotted curve is our analytic approximation to the boost factor (15). The dashed region shows the theoretical expectation for the mass cut-off [2].

tribution). The two-point correlation function of $D(t)$ simply measures the two-point correlation of CDM density, by projecting (12) into the real space, which is then modulated by the annual motion of earth around the sun. This can be done through a simple generalization of (14) to allow for finite separation in real space, and is shown in Fig.(3) for three different cut-offs of the CDM hierarchy, assuming $\rho_{\mathrm{CDM}}=10^{5} \rho_{\text {crit }}$ within the solar system. We thus predict that, depending on the cut-off in the CDM hierarchy, the dark matter detection signal could gradually change by up to a factor of 2 within a ten year period. Potential measurement of this temporal correlation could shed light on the cut-off of the CDM hierarchy, which is directly related to the mass of CDM particles.

To conclude this letter, we will turn to directional dark matter searches, which can directly probe the phase space of the CDM halo. These searches are now underway, and can potentially provide the first telescopes for the rich field of Dark Matter Astronomy. We can use our formalism (Eqs. 10 10 to predict the velocity space correlation function at the solar radius, which can be directly measured if CDM particles are detected in directional searches. This is shown in Fig. (四). For simplicity, we assumed a singular isothermal sphere for the mean phase space density at the solar radius. The dimensionless correlation function is more prominent at higher velocities,

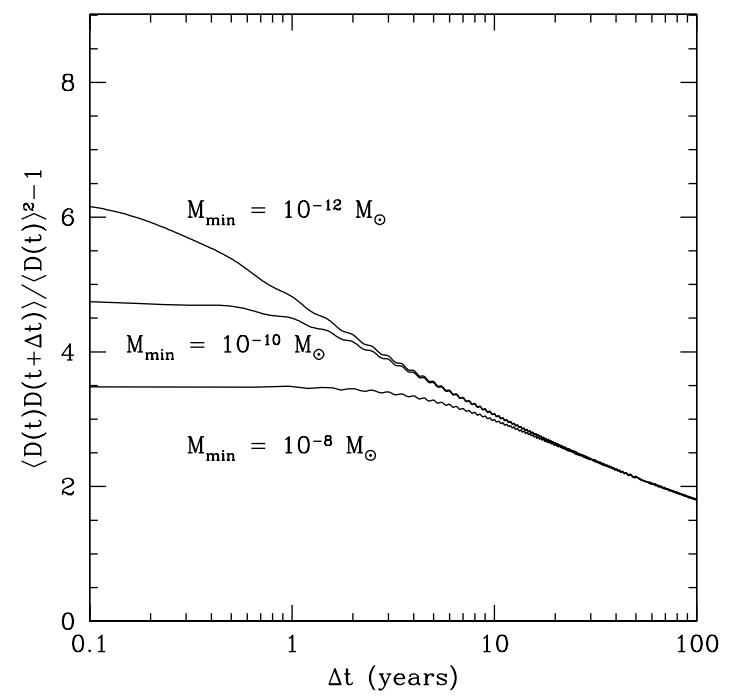

FIG. 3: Temporal correlation function of the dark matter detection signal within the solar system (assuming $\rho_{\mathrm{CDM}}=$ $\left.10^{5} \rho_{\text {crit }}\right)$ for three different cut-offs of the CDM hierarchy.

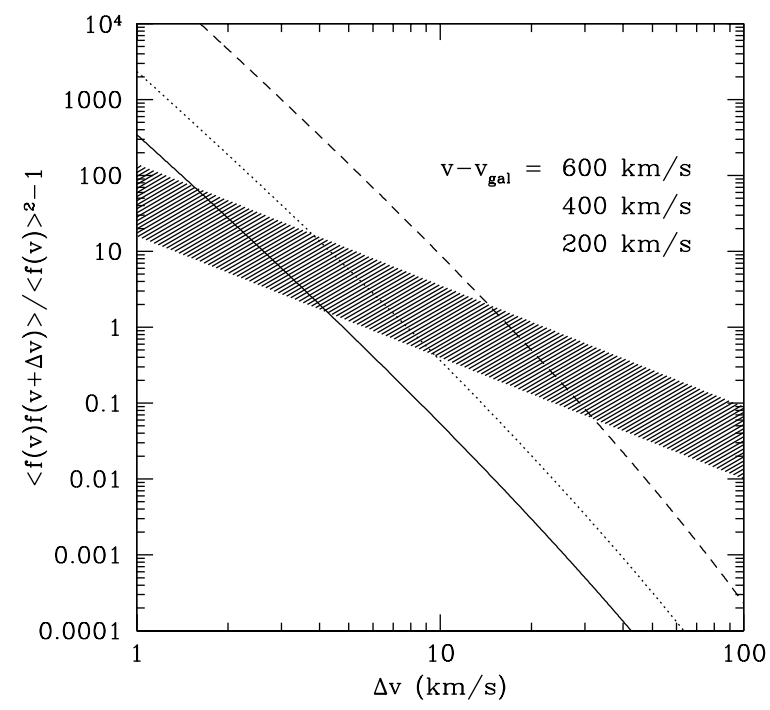

FIG. 4: DM phase space correlation due to bound subhaloes at solar radius. The three lines show velocities: 200, 400, and $600 \mathrm{~km} / \mathrm{s}$ in the Galactic frame. The shaded area shows the expected contribution from unbound substructure [19].

and shows a correlation length of $5-20 \mathrm{~km} / \mathrm{s}$ in the velocity space. For comparison, the shaded area shows the expected level of correlation due to unbound substructure, which is $10-30 \%$ at $\Delta \mathbf{v} \sim 100 \mathrm{~km} / \mathrm{s}$ [20], and scales as $|\Delta \mathbf{v}|^{-1.6}$ 19].

The fact that $B_{\text {sub. }} \sim 2-6$ implies that the local CDM density may be dominated by small subhaloes with random velocities with respect to the Galaxy. This will 
introduce an $\mathcal{O}(1)$ uncertainty in the direction of local dark matter wind (or dipole), which was believed to be the benchmark of directional dark matter searches, or the annual modulation signal. Nevertheless, as we argued above, the richness of structure introduced by the CDM hierarchy leads to novel observables/smoking guns for dark matter searches to aim for.

We thank C.-P. Ma, J. Taylor, M. Vogelsberger and S. White for discussions. NA was partially supported by Perimeter Institute (PI) for Theoretical Physics. Research at PI is supported by the Government of Canada through Industry Canada and by the Province of Ontario through the Ministry of Research \& Innovation. RM thanks the French ANR (OTARIE) for financial support. EB acknowledges support from NASA grant NNG06GG99G.

* Electronic address: nafshordi@perimeterinstitute.ca

[1] P. Gondolo et al., JCAP 0407, 008 (2004).

[2] S. Profumo, K. Sigurdson, and M. Kamionkowski, Phys. Rev. Lett. 97, 031301 (2006).

[3] J. Diemand, M. Kuhlen, and P. Madau, Astrophys. J. 657, 262 (2007).

[4] V. Springel, S. D. M. White, C. S. Frenk, J. F. Navarro, A. Jenkins, M. Vogelsberger, J. Wang, A. Ludlow, and A. Helmi, Nature (London) 456, 73 (2008).

[5] L. E. Strigari, S. M. Koushiappas, J. S. Bullock, and
M. Kaplinghat, Phys. Rev. D75, 083526 (2007).

[6] L. Pieri, G. Bertone, and E. Branchini, Mon. Not. Roy. Astron. Soc. 384, 1627 (2008).

[7] M. Kuhlen, J. Diemand, and P. Madau, Astrophys. J. 686, 262 (2008).

[8] M. Davis and P. J. E. Peebles, , ApJS 34, 425 (1977).

[9] A. J. S. Hamilton, A. Matthews, P. Kumar, and E. Lu, Astrophys. J. 374, L1 (1991).

[10] H. J. Mo, B. Jain, and S. D. M. White, Mon. Not. Roy. Astron. Soc. 276, L25 (1995).

[11] J. A. Peacock and S. J. Dodds, Mon. Not. Roy. Astron. Soc. 280, L19 (1996).

[12] C.-P. Ma and J. N. Fry, ApJL 538, L107 (2000).

[13] R. E. Smith et al. (The Virgo Consortium), Mon. Not. Roy. Astron. Soc. 341, 1311 (2003).

[14] T. Goerdt, O. Y. Gnedin, B. Moore, J. Diemand, and J. Stadel, Mon. Not. Roy. Astron. Soc. 375, 191 (2007).

[15] J. E. Gunn and I. Gott, J. Richard, Astrophys. J. 176, 1 (1972).

[16] N. Afshordi and R. Cen, Astrophys. J. 564, 669 (2002).

[17] J. M. Bardeen, J. R. Bond, N. Kaiser, and A. S. Szalay, Astrophys. J. 304, 15 (1986).

[18] D. J. Eisenstein and W. Hu, Astrophys. J. 496, 605 (1998).

[19] N. Afshordi, R. Mohayaee, and E. Bertschinger, Phys. Rev. D 79, 083526 (2009).

[20] M. Vogelsberger, A. Helmi, V. Springel, S. D. M. White, J. Wang, C. S. Frenk, A. Jenkins, A. Ludlow, and J. F. Navarro, MNRAS 395, 797 (2009). 